PAPER

\title{
Interictal language functions in temporal lobe epilepsy
}

\author{
L Bartha, T Benke, G Baver, E Trinka
}

J Neurol Neurosurg Psychiatry 2005;76:808-814. doi: 10.1136/jnnp.2004.045385

Objective: To evaluate interictal language functions in patients with medically intractable left and right sided mesial temporal lobe epilepsy (TLE).

See end of article for authors' affiliations ......................

Correspondence to: Dr Lisa Bartha, Innsbruck Medical University, Clinical Department of Neurology, Anichstraße 35, A-6020 Innsbruck, Austria; lisa.bartha@uibk. ac.at

Received 10 May 2004 Revised version received 6 October 2004 Accepted 6 October 2004
Methods: Spontaneous speech, language comprehension, confrontation naming, repetition, reading, writing, and word fluency were examined in 12 patients with left sided TLE and 11 patients with right sided TLE.

Results: Four patients out of 23 displayed language deficits in more than one language domain. Three further patients exhibited isolated language deficits. Linguistic deficits were observed in both left TLE and right TLE. In quantitative analyses left and right TLE only differed in spontaneous speech $(p=0.02)$; no difference was found in other language functions, laterality quotient of Wada test, or overall IQ. Qualitative error analysis of object naming, however, showed typical errors associated only with left TLE. Patients with linguistic deficits were older at testing compared to patients without linguistic deficits $(p=0.003)$, whereas other factors including side of TLE, handedness, educational level, age at epilepsy onset, and duration of epilepsy did not differ between groups.

Conclusions: Possible explanations for these findings include neuronal cell loss and deafferentiation in cortical areas, and disruption of the basal temporal language area pathways. Our study suggests that some patients with chronic mesial TLE exhibit linguistic deficits when specifically tested, and underlines the need to routinely investigate linguistic functions in TLE.
$\mathrm{T}$ he classical neural substrates of language functions include various sites in the dominant temporal neocortex, the frontal cortex, and the inferior parietal cortex. ${ }^{1}$ Thus, mesiobasal structures do not belong to the classical language areas. Therefore, it is not surprising that comparatively little has been reported on the linguistic abilities of patients with mesial temporal lobe epilepsy (TLE), although much is known about memory functions in these patients. ${ }^{2} \mathrm{~A}$ survey of the literature shows that confrontation naming is usually the sole language function tested in TLE, and is reported to be reduced in most investigations. ${ }^{3-6}$ Studies comparing naming in left versus right TLE presented differing findings. Whereas some researchers could not detect any significant differences in comparing interictal visual naming performance in left versus right $\mathrm{TLE}^{7}{ }^{7}$ others found confrontation naming in left TLE worse than in right TLE. ${ }^{6-10}$ However, many studies were interested in differences between patient groups but did not compare the patients' naming performances to normal controls. ${ }^{6}{ }^{10}$ Therefore, it often remains unclear whether or not these patients were impaired in their naming performance. Furthermore, information about language comprehension, discourse production, repetition, and reading is sparse, and findings are divergent. ${ }^{11-14}$ To our knowledge, to date there are no reports on spontaneous speech or writing abilities in TLE. Thus, studies have very rarely administered tests tapping different language domains in order to provide a linguistic profile of patients with TLE.

This study aims to assess linguistic functions in patients with unilateral TLE. Twenty three patients with medically intractable TLE were investigated prospectively with a comprehensive neurolinguistic test battery.

\section{METHODS}

Twenty three consecutive patients with medically refractory TLE were recruited between 2000 and 2001 during a presurgical evaluation program run by the Universitätsklinik für Neurologie, Innsbruck, Austria. All patients underwent detailed clinical and neurological examination, high resolution magnetic resonance imaging (MRI), prolonged video-EEG monitoring including detailed analysis of seizure semiology, ictal EEG, and quantitative spike distribution, a comprehensive neuropsychological examination, and Wada test when feasible. Inclusion criteria were: (i) unilateral seizure onset zone evaluated by ictal EEG; (ii) unilateral interictal EEG with a left to right spike distribution not less than $90 \%$ to $10 \%$ with or without unilateral slowing over the temporal lobe; and (iii) an epileptogenic lesion, visualised by high resolution MRI in the mesial temporal areas concordant with the side of seizure onset. We excluded patients with: (i) ictal EEG changes or independent seizure onset from both temporal lobes; (ii) interictal discharges over both temporal lobes with a left to right distribution less than $90 \%$ to $10 \%$; and (iii) evidence of bitemporal structural changes (for example, bitemporal hippocampal atrophy) or extratemporal lesions on MRI.

Language was assessed using the Aachener Aphasie Test $(\mathrm{AAT}),{ }^{15}$ the Innsbrucker Benenntest (IBT), ${ }^{16}$ and an adapted, German version of a word fluency test developed by Vilkki and Holst. ${ }^{17}$ Language testing was performed more than 7 days after the last reported seizure. Spontaneous speech was evaluated with the AAT using a semi-structured interview. This interview contains questions concerning the patient's profession, family, hobbies, and sensitivities and lasts approximately $10 \mathrm{~min}$. Subsequent to this interview, the tester rated communicative behaviour, articulation, prosody and semantic, syntactic, and phonemic aspects of spontaneous speech by means of a given six-level scale. Language comprehension was evaluated using the AAT Token Test and the AAT comprehension test, which requires verbal and written stimuli to be matched to one of four pictures. Production of single words, compounds, colour words, and

Abbreviations: AAT, Aachener Aphasie Test; IBT, Innsbrucker Benenntest; MRI, magnetic resonance imaging; TLE, temporal lobe epilepsy 
sentences was tested with the corresponding AAT naming subtest, which consists of 40 pictures of objects, colours, and scenes. In addition, the IBT was performed, which is a confrontation naming test with 25 living and 25 non-living objects which range from low to high frequency of appearance in the everyday environment. ${ }^{16}$ In this test, semantic and phonemic cues are proposed in case of word finding difficulties to evaluate the cause of the naming deficit. Reading and writing of words and sentences was assessed using the corresponding subtests of the AAT. Furthermore, composition of words and sentences from dictation with different given letter tokens was tested. Repetition was investigated employing the AAT repetition subtest which contains 50 items including sounds, one syllable words, foreign words, compounds, and sentences.

Additionally, a word fluency test was used which requests the patient to name as quickly as possible (i) 20 different animals, (ii) 10 different words beginning with the letter " $\mathrm{S}$ ", and (iii) animals and S words alternately, 10 out of each category. ${ }^{17}$ Each subtest of this word fluency task was stopped after $3 \mathrm{~min}$ in case of null response and maximum time limit was 10 min each. Evaluation of the patient's performance in this test includes time measurement and possible errors, for example, perseveration of items (words previously produced in the word fluency task) and stuck in sets (inflexibility on category alternation). This test was added to the present study because word fluency tasks are widely used in examining patients with epilepsy; however, as these tests assess not only language production but also psychomotor speed and cognitive flexibility, ${ }^{18}$ we did not consider this test a part of the language battery.

The AAT Token Test, the IBT Naming Test, and the word fluency test have normative data from different age groups ranging from 20 to over 70 years. Therefore, in these tests we were able to compare patients' performances with norms from the corresponding age group. A deficit or impairment in each language domain was established when performance in the overall, additive score of a test was below the third percentile or more than 2 standard deviations (SD) below the standardisation group's mean score. Impairment on a single subtest of a test did not equal a deficit in that language domain in this study.

\section{RESULTS}

\section{Patients}

High resolution MRI revealed unilateral hippocampal sclerosis in 21 patients, a tumour in the mesial temporal lobe in one patient (patient $\mathrm{F}$ ), and a dysplastic lesion in the mesial temporal lobe in one patient $(\mathrm{H})$. The lateral temporal neocortices were normal in all patients on visual inspection. Edinburgh Handedness Inventory laterality quotients ${ }^{19}$ revealed a strong right handedness in all patients. The Wada test laterality quotient was calculated by the formula [(right-left)/(right+left)*(score of better performance)/ (overall score)] and showed left dominant language functions in 21 patients and bilateral lanyguage localisation in one patient (patient C). A selective amygdalo-hippocampectomy was performed in 21 patients and revealed Ammon's horn sclerosis in 19 patients, focal cortical dysplasia in one patient $(\mathrm{H})$, and an astrocytoma grade II in one patient $(\mathrm{F})$. Demographical data of the patients are shown in table 1.

\section{Neurolinguistic findings}

Language assessment showed fluent, well articulated spontaneous speech without semantic or phonemic paraphasias, neologisms, or perseverations as well as intact situative language comprehension in all patients. Two patients (patients E, G) exhibited word finding deficits, that is, the failure to produce a name, hesitations, and searching behaviour in spontaneous speech that impaired communicative competence in both of them. Formal language testing revealed intact language comprehension in 20 patients with three of them (patients E, F, M) showing minor errors in single subtests of language comprehension, and three patients (patients B, I, Q) displaying moderate deficits in different aspects of language comprehension. Errors mostly appeared in choosing semantic foils in the auditory and written comprehension task $(82 \%$ of errors in the AAT language comprehension test) and with increasing syntactic complexity in the Token Test $(67 \%$ of errors in the Token Test). No patient showed phonemic comprehension errors in pointing to foils phonologically related to the target word. In confrontation naming, patients' performance was highly dependent on the type of stimuli. Whereas the production of high frequency nouns and colour words did not show significant deficits, naming of low frequent objects and objects with compound names yielded significant deficits in six patients (patients B, C, E, G, M, Q). Errors in naming objects (AAT compounds and IBT naming) consisted of word finding deficits (49\%), naming of objects visually and semantically related to the target (for example, patient Q: "violin" instead of guitar; overall 27\%), semantic paraphasias (for example, patient G: "fish" instead of crocodile; overall $18 \%)$, and visuoperceptive misnamings (6\%), while syntactic or phonemic errors did not occur. In addition, patient $G$ revealed word finding deficits and semantic paraphasias in naming situations. In the IBT naming test, $55 \%$ of errors were produced when naming living objects (27 v 22 errors), thus, there was a trend towards worse naming of living compared to non-living objects, but no significant category specific naming effect was found. When patients exhibited word finding deficits, semantic cues helped in $9.5 \%$ and phonemic cues in $40.9 \%$ of cases.

Two patients (patients C, Q) showed some minor errors in repetition of sentences and one patient (patient C) in composition of words. Nevertheless, overall repetition performance and written language performance were intact in all patients. In sum, interpreting the overall scores of the language subtests, four patients (patients B, E, G, Q) exhibited linguistic deficits in more than one language domain, and three patients (patients C, I, M) displayed isolated linguistic deficits in naming $(\mathrm{C}, \mathrm{M})$ and language comprehension (I), respectively.

Furthermore, alternating word fluency, not considered a part of the language battery, showed a profound impairment in nearly all patients; only patients $\mathrm{K}, \mathrm{T}$, and $\mathrm{W}$ showed intact abilities in all three fluency tasks. Eighteen patients demonstrated significantly reduced word generation, four of whom (patients E, G, M, U) were unable to produce 20 items in $10 \mathrm{~min}$. Six patients furthermore displayed numerous perseverations during word generation (patients $\mathrm{B}, \mathrm{D}, \mathrm{F}, \mathrm{Q}, \mathrm{S}, \mathrm{V}$ ), two patients were not able to alternate correctly between the tasks (patients $\mathrm{H}, \mathrm{S}$ ), and two patients broke rules by producing categories other than those demanded (patients B, $S)$. The results of the neurolinguistic tests are summarised in table 2 .

Overall, four patients out of 23 (patients B, E, G, Q) displayed language deficits in more than one language domain (excluding word fluency). In these patients, the severity and combination of deficits varied, but linguistic deficits never affected phonological or syntactic aspects of language. Furthermore, three patients (patients C, I, M) exhibited isolated language deficits.

\section{Statistical analysis of demographical and neurolinguistic findings}

We were interested whether the patients showing language deficits differed demographically compared to patients 
Table 1 Patient data

\begin{tabular}{|c|c|c|c|c|c|c|c|c|c|c|c|c|c|c|c|}
\hline Patient & Age & Sex & $\begin{array}{l}\text { Hand- } \\
\text { edness } \\
\text { LQ }\end{array}$ & $\begin{array}{l}\text { Over- } \\
\text { all } \\
I^{*}\end{array}$ & $\begin{array}{l}\text { Age at } \\
\text { seizure } \\
\text { onset }\end{array}$ & Aura type & $\begin{array}{l}\text { Febrile } \\
\text { seizures }\end{array}$ & Medication & iiEEG & iEEG & MRI & $\begin{array}{l}\text { PET/ } \\
\text { SPECT }\end{array}$ & $\begin{array}{l}\text { WADA } \\
\text { LQ }\end{array}$ & $\begin{array}{l}\text { Type of } \\
\text { surgery }\end{array}$ & Pathology \\
\hline A & 39 & $\mathrm{~F}$ & 90 & 128 & 33 & Psychic & No & TPM & LT & LT & HS L & LT & 0.84 & AHE L & AHS \\
\hline B & 45 & $\mathrm{~F}$ & 100 & 81 & 10 & Epigastric & Yes & PHE & LT & LT & HS L & LT & 0.84 & AHE L & AHS \\
\hline C & 49 & $M$ & 100 & 76 & 17 & $\begin{array}{l}\text { Epigastric, } \\
\text { sensory }\end{array}$ & No & $\begin{array}{l}\text { PRM, VPA, } \\
\text { CLB }\end{array}$ & LT & LT & HS L & LT & 0.13 & AHE L & AHS \\
\hline D & 34 & $M$ & 100 & 111 & 4 & Psychic & No & CBZ & LT & LT & HS L & $\mathrm{RT}$ & 0.63 & AHE L & AHS \\
\hline$E$ & 51 & $F$ & 20 & 70 & 5 & $\begin{array}{l}\text { Epigastric, } \\
\text { sensory }\end{array}$ & Yes & CBZ, TPM & LT & LT & HS L & LT & 0.40 & AHE L & AHS \\
\hline $\mathrm{F}$ & 40 & $M$ & 50 & 70 & 15 & $\begin{array}{l}\text { Epigastric, } \\
\text { vegetative }\end{array}$ & No & $\mathrm{CBZ}, \mathrm{PB}$ & LT & LT & Tumour L & ND & 0.69 & AHE L & $\begin{array}{l}\text { Astro- } \\
\text { cytoma II }\end{array}$ \\
\hline G & 41 & $M$ & 100 & 86 & 18 & Epigastric & Yes & $C B Z$ & LT & LT & HS L & LT & 0.69 & AHE L & AHS \\
\hline $\mathrm{H}$ & 46 & $M$ & 100 & 111 & 11 & Epigastric & No & TPM & LT & LT & DYSPL L & ND & 0.77 & AHE L & FCD \\
\hline I & 52 & $\mathrm{~F}$ & 100 & 105 & 30 & Psychic & No & $\mathrm{PB}, \mathrm{CLB}$ & LT & LT & HS L & ND & 0.80 & AHE L & AHS \\
\hline J & 35 & $\mathrm{~F}$ & 100 & 76 & 2 & Psychic & No & LTG, OXC & LT & LT & $\mathrm{HS} \mathrm{L}$ & LT & 0.67 & AHE L & AHS \\
\hline K & 40 & $M$ & 0 & 118 & 5 & Epigastric & Yes & CBZ & LT & LT & HS L & LT & ND & None & \\
\hline $\mathrm{L}$ & 30 & $\mathrm{~F}$ & 100 & 95 & 16 & Psychic & Yes & CBZ, VPA & LT & LT & $\mathrm{HS} \mathrm{L}$ & LT & 0.80 & None & \\
\hline$M$ & 47 & $M$ & 100 & 86 & 26 & $\begin{array}{l}\text { Vegetative, } \\
\text { psychic }\end{array}$ & No & CBZ & RT & RT & $\mathrm{HS} \mathrm{R}$ & RT & 1.00 & AHE R & AHS \\
\hline$N$ & 45 & $\mathrm{~F}$ & 100 & 112 & 25 & None & No & CBZ & RT & RT & HS R & ND & 0.69 & AHE R & AHS \\
\hline 0 & 48 & $M$ & 100 & 128 & 19 & $\begin{array}{l}\text { Sensory, } \\
\text { vegetative }\end{array}$ & Yes & VPA, CBZ & RT & RT & HS R & LT & 1.00 & AHE R & AHS \\
\hline$P$ & 62 & $M$ & 80 & 112 & 21 & $\begin{array}{l}\text { Epigastric, } \\
\text { vegetative }\end{array}$ & No & TPM, CLB & RT & RT & HS R & RT & 0.33 & AHE R & AHS \\
\hline$Q$ & 51 & $\mathrm{~F}$ & 100 & 74 & 8 & None & No & $\mathrm{PB}, \mathrm{CLB}$ & RT & RT & HS R & ND & 0.86 & AHE R & AHS \\
\hline $\mathrm{R}$ & 40 & $M$ & 100 & 93 & 13 & Epigastric & Yes & CBZ & RT & RT & HS R & ND & 0.42 & AHE R & AHS \\
\hline$S$ & 44 & $\mathrm{~F}$ & 80 & 90 & 13 & $\begin{array}{l}\text { Strange } \\
\text { feeling }\end{array}$ & No & TPM, CLB & RT & RT & HS R & LT & 0.47 & AHE R & AHS \\
\hline $\mathrm{T}$ & 42 & $\mathrm{~F}$ & 100 & 97 & 8 & $\begin{array}{l}\text { Epigastric, } \\
\text { gustatory }\end{array}$ & No & $C B Z$ & RT & RT & HS R & RT & 0.48 & AHE R & AHS \\
\hline U & 45 & $M$ & 60 & 98 & 7 & Epigastric & No & CBZ, TPM & RT & RT & HS R & RT & 0.00 & AHE R & AHS \\
\hline V & 37 & $\mathrm{~F}$ & 80 & 95 & 1 & Fear & Yes & CBZ, LTG & RT & RT & HS R & RT & 1.00 & AHE R & AHS \\
\hline W & 31 & $M$ & 100 & 98 & 13 & None & No & VGB, VPA & RT & RT & HS R & ND & 0.85 & AHE R & AHS \\
\hline Mean & 43.2 & & 80.0 & 96.1 & 13.9 & & & & & & & & & & \\
\hline SD & 7.4 & & 40.8 & 17.4 & 8.8 & & & & & & & & & & \\
\hline
\end{tabular}

*Overall intelligence was measured with the Lehrl Mehrfach Wortschatz Intelligenz Test. ${ }^{46}$

AHE, selective amygdalo-hippocampectomy; AHS, Ammon's horn sclerosis; BT, bitemporal; CBZ, carbamazepine; CLB, clobazam; DYSPL, dysplasia; FCD, focal cortical dysplasia (Taylor type); HS, hippocampal sclerosis; iEEG, ictal EEG; iiEEG, interictal EEG; L, left; LQ, laterality quotient; LT, left temporal; LTG, lamotrigine; ND, not done; OXC, oxcarbazepine; PB, phenobarbital; PHE, phenytoin; PRM, primidone; R, right; RT, right temporal; TPM, topiramate; VGB, vigabatrin; VPA, valproate.

without linguistic impairment. We therefore divided the patients into two groups according to the presence or absence of a linguistic impairment which was established when performance fell below the third percentile or more than 2 SD below the mean score. Mann-Whitney $U$ test revealed that patients with language deficits (patients $\mathrm{B}, \mathrm{C}, \mathrm{E}$, G, I, M, Q) were significantly older at the time of neurolinguistic assessment compared to patients without language deficits (mean age (years) 41.1, SD 7.7, and mean age 48.0, SD 4.0, respectively; $\mathrm{p}=0.009$ ). However, they did not differ with regard to side of TLE, handedness, educational level, age at epilepsy onset, or duration of epilepsy. Non-parametric correlation revealed that overall naming performance correlated with the overall IQ of the patients (Spearman's $\rho \mathrm{p}=0.002$ ). In addition, language comprehension negatively correlated with the laterality index derived from the Wada test $(p=0.004)$, that is, patients with deficits in language comprehension showed more lateralised language functions in the Wada test. Other scores of language subtests did not correlate with demographic factors.

We were furthermore interested whether patients with left TLE differed in their language profile from those with right TLE. Mann-Whitney $U$ test revealed that the results of only one test, that is, spontaneous speech, were significantly worse in patients with left TLE $(p=0.02)$; the results of other language function tests, the laterality quotient of the Wada test, and overall IQ did not differ significantly between the two groups. Consequently, a nonparametric Spearman's $\rho$ correlation revealed that the side of TLE significantly correlated with performance in spontaneous speech $(\mathrm{p}=0.02)$. However, if the analysis was based on qualitative rather than quantitative results, a difference in naming between the two groups became apparent. Error analysis in naming objects revealed that only patients with left sided TLE produced semantic paraphasias, whereas patients with right sided TLE did not show this error type (fig 1). The incidence of other error types, that is, word finding difficulties, errors visually and semantically related to the target, and visuoperceptive errors, did not differ between the groups.

In order to detect typical clusters of language deficits in our patients, we performed a non-parametric Spearman's $\rho$ correlation. We found that spontaneous speech significantly correlated with naming of objects $(p=0.002)$ and written language abilities $(p=0.001)$. Overall naming performance furthermore correlated with performance in the Token Test $(\mathrm{p}=0.04)$ and written language abilities $(\mathrm{p}=0.04)$. Repetition and word fluency did not correlate with any other linguistic performance.

In sum, linguistic deficits were observed in both left TLE and right TLE; in a quantitative analysis these groups only differed significantly in spontaneous speech. A qualitative error analysis of object naming yielded significant differences between these two groups. Furthermore, patients with linguistic deficits showed a significant higher age at testing, while other factors including age at epilepsy onset and duration of epilepsy, did not differ between groups and did not correlate with language deficits. However, we have to stress the limitations of statistical power due to the relatively small sample size in this study. 


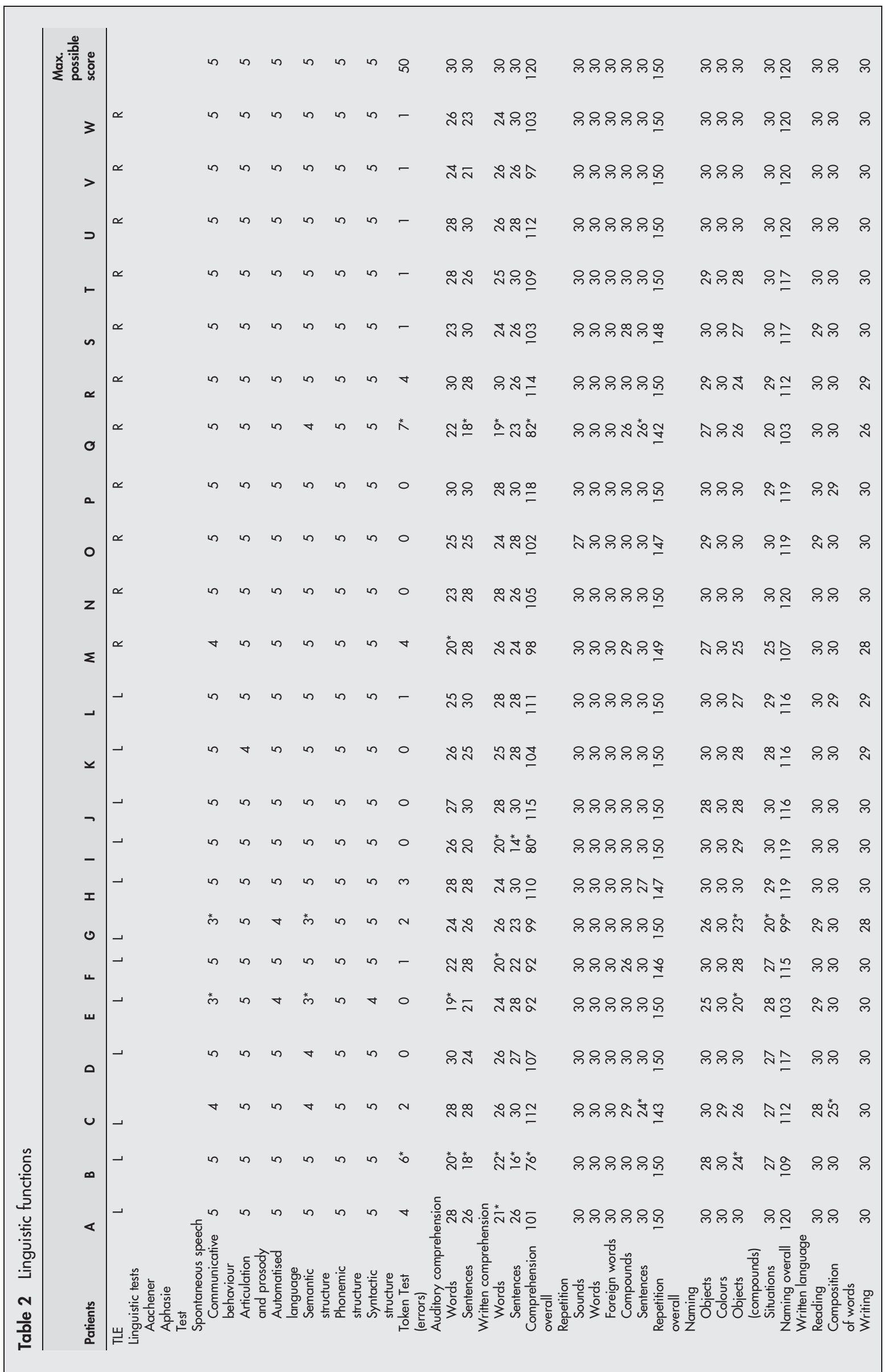



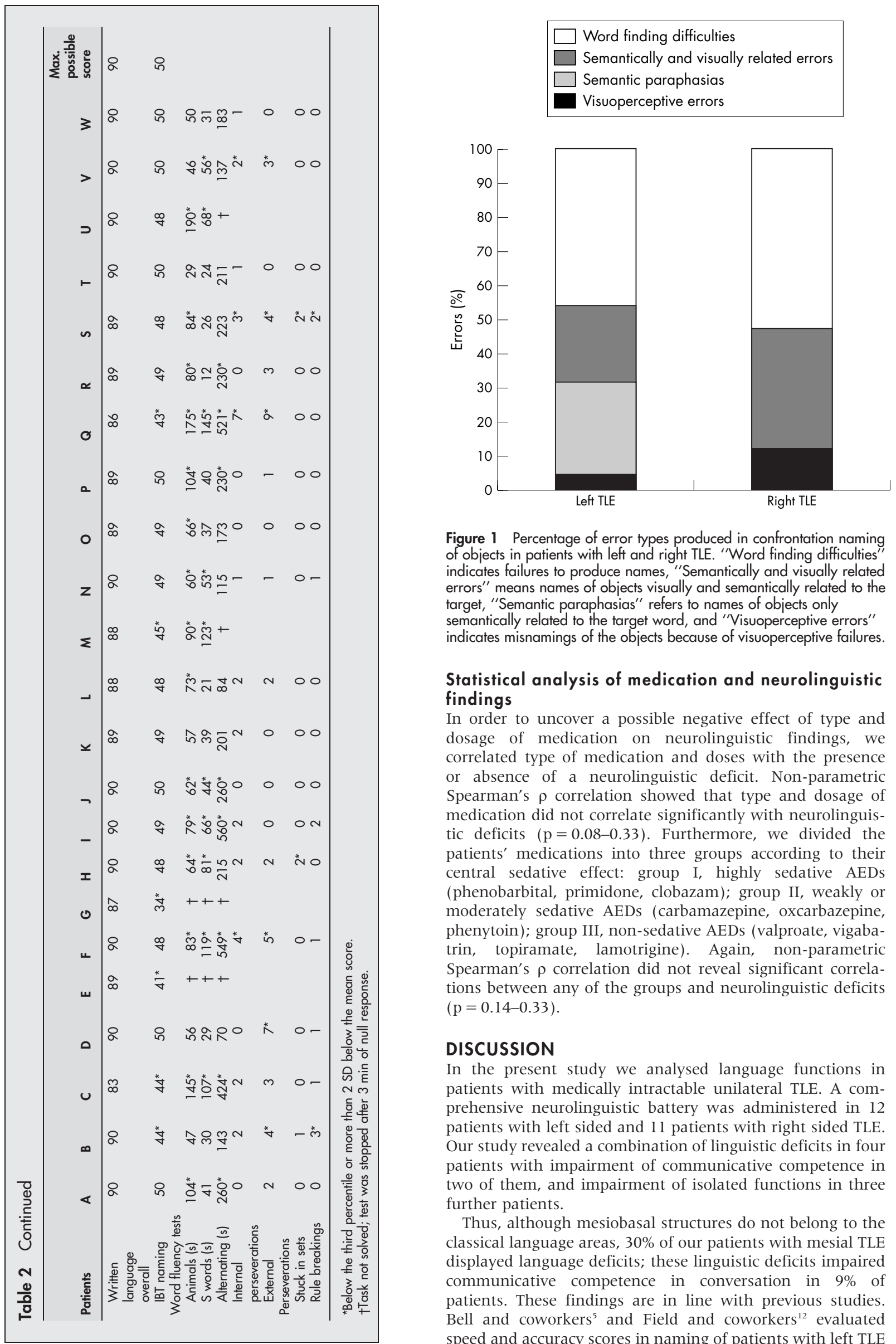

Figure 1 Percentage of error types produced in confrontation naming of objects in patients with left and right TLE. "Word finding difficulties" indicates failures to produce names, "Semantically and visually related errors" means names of objects visually and semantically related to the target, "Semantic paraphasias" refers to names of objects only semantically related to the target word, and "Visuoperceptive errors" indicates misnamings of the objects because of visuoperceptive failures.

\section{Statistical analysis of medication and neurolinguistic findings}

In order to uncover a possible negative effect of type and dosage of medication on neurolinguistic findings, we correlated type of medication and doses with the presence or absence of a neurolinguistic deficit. Non-parametric Spearman's $\rho$ correlation showed that type and dosage of medication did not correlate significantly with neurolinguistic deficits $(p=0.08-0.33)$. Furthermore, we divided the patients' medications into three groups according to their central sedative effect: group I, highly sedative AEDs (phenobarbital, primidone, clobazam); group II, weakly or moderately sedative AEDs (carbamazepine, oxcarbazepine, phenytoin); group III, non-sedative AEDs (valproate, vigabatrin, topiramate, lamotrigine). Again, non-parametric Spearman's $\rho$ correlation did not reveal significant correlations between any of the groups and neurolinguistic deficits $(\mathrm{p}=0.14-0.33)$.

\section{DISCUSSION}

In the present study we analysed language functions in patients with medically intractable unilateral TLE. A comprehensive neurolinguistic battery was administered in 12 patients with left sided and 11 patients with right sided TLE. Our study revealed a combination of linguistic deficits in four patients with impairment of communicative competence in two of them, and impairment of isolated functions in three further patients.

Thus, although mesiobasal structures do not belong to the classical language areas, $30 \%$ of our patients with mesial TLE displayed language deficits; these linguistic deficits impaired communicative competence in conversation in $9 \%$ of patients. These findings are in line with previous studies. Bell and coworkers ${ }^{5}$ and Field and coworkers ${ }^{12}$ evaluated speed and accuracy scores in naming of patients with left TLE 
and healthy adults and found naming speed and accuracy worse in patients with TLE than in controls. Hermann and coworkers ${ }^{9}$ evaluated repetition, oral spelling, comprehension, reading, and oral word association and reported language deficits in both left and right TLE; however, information about the type and extent of these deficits is not available. Three studies were interested in discourse abilities in TLE and two of them found that the patients performed significantly worse in a narrative discourse task that required description of the sequence of events. ${ }^{10-12}$

Reasons for linguistic deficits found in patients with TLE are not completely clear, and different hypotheses can be formulated. First, multiple regions of the brain, which may also include the left perisylvian areas critical for language, may be subject to neuronal cell loss and deafferentiation in chronic TLE. This is supported by studies demonstrating that there are widespread structural deficits in patients with chronic TLE. ${ }^{20-22}$ In accordance with this, in patients with TLE, hypometabolism was not only found in mesiobasal structures but also in neocortical areas including the lateral temporal neocortex ipsi- and contralateral to the hippocampal atrophy and sclerosis. ${ }^{23-26}$ Furthermore, neuropsychological studies of patients with mesial TLE revealed cognitive deficits associated with frontal, temporal, and parietal areas. ${ }^{27-29}$ These findings are in accordance with previous reports and the present study which demonstrate linguistic deficits associated with neocortical dysfunction in chronic left and right TLE.

Second, disruption of pathways to and from the parahippocampal and fusiform gyri may be possible mechanisms underlying the language deficits observed in our study. There is some evidence from lesion, stimulation, and neuroimaging studies of a basal temporal language area. Lüders and coworkers showed that electrical stimulation to the fusiform gyrus of the language dominant hemisphere at high intensity caused a transient aphasia with both language comprehension and production deficits, whereas electrical stimulation at lower intensity induced anomia. ${ }^{30}{ }^{31}$ Surgical resection of left basal temporal areas in patients with TLE is reported to risk naming deficits. ${ }^{27}{ }^{32}$ Functional imaging studies underline these findings as they found the left fusiform gyrus active during picture naming, ${ }^{33}$ the linguistic function also found to be the most impaired in our study.

Third, hippocampal sclerosis and chronic TLE may affect the anatomical representation of cognitive functions and may cause a reorganisation of the language network. Recent studies suggest that the hippocampal formation plays a role in some aspects of language processing in healthy individuals. ${ }^{34-36}$ However, cognitive models developed in studies with normal subjects may not apply to patients with chronic TLE. In fact, studies using the Wada test, transcranial Doppler sonography, or functional MRI have demonstrated a more heterogeneous pattern of language lateralisation and language localisation in patients with TLE compared to normals. ${ }^{37-39}$ It may therefore be possible that the contribution of the hippocampal formation to language processing is variable, and that the hippocampus participates in language processing in some, but not all, patients with TLE. Thus, hippocampal atrophy and sclerosis may affect language functions in some patients, depending on their intrahemispheric language organisation. Furthermore, to some extent language reorganisation even seems to prevent patients from experiencing language deficits, as our study shows that the language laterality index derived from the Wada test significantly correlates with language comprehension, and that auditory and visual comprehension of words and sentences is better preserved in patients with language functions less strongly lateralised to the left hemisphere.
We were also interested in the pattern of language deficits in patients with TLE. Many studies on TLE apply confrontation naming or word fluency tests for measuring overall language functioning. However, clusters of deficits were found in some, but not all, patients. Thus, a general impairment in language functioning should not be presumed on the basis of a deficit in a single language ability such as object naming. Furthermore, word fluency, not considered a part of the language battery used in our study, was reduced in nearly all patients and did not correlate with any other linguistic function. These findings are in line with previous reports on word fluency deficits in patients with TLE. ${ }^{40}$ These deficits can hypothetically be interpreted as an inefficiency in initiating, maintaining, and switching systematic search and retrieval strategies. This hypothesis is indirectly supported by reports of set shifting difficulties and perseverations on the Wisconsin Card Sorting Test (typically associated with executive deficits accompanying frontal pathology) in patients with TLE. ${ }^{41}{ }^{42}$ Thus, distant frontal pathophysiology might underlie decreased word fluency in our patients. However, word fluency deficits found in TLE patients may also reflect dysfunctioning of a basal temporal language area involved in semantic language memory, as discussed above. There is some evidence in the literature that disruption of semantic networks underlies deficient verbal fluency not only in left but also in right TLE. Joanette and Goulet ${ }^{43}$ showed that right hemisphere damage can impair semantic fluency, and Lifrak and Novelly ${ }^{44}$ and Martin et $a l^{45}$ reported deficits in word fluency following right temporal lobectomy.

Therefore, we assume that naming or word fluency tasks on their own are not appropriate measurements for language functions in TLE. Thus, in order to obtain a reliable profile of language functions and language deficits in patients with TLE, it is necessary to evaluate different language domains including spontaneous speech, language comprehension, naming, reading, writing, and word fluency. ${ }^{15}$

Our study has some limitations which have to be stressed. First, the relatively small sample size limits the statistical power of our study. Therefore, the lack of association between the side of TLE and linguistic deficits might be due to the reduced statistical power (spontaneous speech was the only language function significantly worse in the group of patients with left TLE). Second, patient interviews were not video taped, so we were unable to perform blinded inter-rater reliability studies.

In sum, the present study shows that some patients with chronic mesial TLE exhibit linguistic deficits when specifically tested, independent of the side of epilepsy. Our findings suggest that greater attention should be directed towards linguistic deficits in patients with temporal lobe epilepsy.

\section{Authors' affiliations}

L Bartha, T Benke, G Bauer, E Trinka, Clinical Department of Neurology, Innsbruck Medical University, Anichstraße 35, A-6020 Innsbruck,

Austria

Competing interests: none declared

\section{REFERENCES}

1 Ojemann GA, Sutherling WW, Lesser RP, et al. Cortical stimulation. In: Engel J, ed. Surgical treatment of the epilepsies. New York: Raven Press, 1993:339-414.

2 Helmstaedter $\mathrm{C}$. Effects of chronic epilepsy on declarative memory systems. Prog Brain Res 2002;135:439-53.

3 Sawrie SM, Martin RC, Gilliam FG, et al. Visual confrontation naming and hippocampal function: a neural network study using quantitative $(1) \mathrm{H}$ magnetic resonance spectroscopy. Brain 2000;123:770-80.

4 Hamberger MJ, Tamny TR. Auditory naming and temporal lobe epilepsy. Epilepsy Res 1999;35:229-43.

5 Bell BD, Seidenberg M, Hermann BP, et al. Visual and auditory naming in patients with left or bilateral temporal lobe epilepsy. Epilepsy Res 2003;55:29-37. 
6 Mayeux R, Brandt J, Rosen J, et al. Interictal memory and language impairment in temporal lobe epilepsy. Neurology 1980;30:120-5.

7 O'Shea MF, Saling MM, Bladin PF, et al. Does naming contribute to memory self-report in temporal lobe epilepsy? J Clin Exp Neuropsychol 1996:18:98-109

8 Saykin AJ, Stafiniak P, Robinson $\sqcup$, et al. Language before and after temporal lobectomy: specificity of acute changes and relation to early risk factors. Epilepsia 1995;36:1071-7

9 Hermann BP, Seidenberg M, Haltiner A, et al. Adequacy of language function and verbal memory performance in unilateral temporal lobe epilepsy. Cortex 1992;28:423-33.

10 Howell RA, Saling MM, Bradley DC, et al. Interictal language fluency in temporal lobe epilepsy. Cortex 1994;30:469-78.

11 Bell B, Dow C, Watson ER, et al. Narrative and procedural discourse in temporal lobe epilepsy. J Int Neuropsychol Soc 2003;9:733-9.

12 Field SJ, Saling MM, Berkovic SF. Interictal discourse production in temporal lobe epilepsy. Brain Lang 2000;74:213-22.

13 Giovagnoli AR. Verbal semantic memory in temporal lobe epilepsy. Acta Neurol Scand 1999:99:334-9.

14 Breier JI, Fletcher JM, Wheless JW, et al. Profiles of cognitive performance associated with reading disability in temporal lobe epilepsy. J Clin Exp Neuropsychol 2000;22:804-16.

15 Huber W, Poeck K, Weniger D, et al. Aachener Aphasie Test. Göttingen: Hogrefe, 1983.

16 Bartha L, Delazer M, Benke T. Die Entwicklung des Innsbrucker Benenntest (IBT). Z Neuropsychol 2001;12:348.

17 Vilkki J, Holst P. Speed and flexibility on word fluency tasks after focal brain lesions. Neuropsychologia 1994;32:1257-62.

18 Lezak MD. Neuropsychological assessment. New York: Oxford University Press, 1995.

19 Oldfield RC. The assessment and analysis of handedness: the Edinburgh inventory. Neuropsychologia 1971;9:97-113.

20 Liu RS, Lemieux L, Bell GS, et al. Progressive neocortical damage in epilepsy. Ann Neurol 2003;53:312-24.

21 Spencer SS, Williamson PD, Spencer DD, et al. Human hippocampal seizure spread studied by depth and subdural recording: the hippocampal commissure. Epilepsia 1987;28:479-89.

22 Moran NF, Lemieux L, Kitchen ND, et al. Extrahippocampal temporal lobe atrophy in temporal lobe epilepsy and mesial temporal sclerosis. Brain 2001; 124:167-75

23 Semah F. Temporoporal metabolic abnormalities in temporal lobe epilepsies. Epileptic Disord 2002;4:S41-9.

24 Hammers A, Koepp MJ, Labbe C, ef al. Neocortical abnormalities of [11C] flumazenil PET in mesial temporal lobe epilepsy. Neurology 2001;56:897-906

25 Rubin E, Dhawan V, Moeller JR, et al. Cerebral metabolic topography in unilateral temporal lobe epilepsy. Neurology 1995;45:2212-23.

26 Sackellares JC, Siegel GJ, Abou-Khalil BW, et al. Differences between lateral and mesial temporal metabolism interictally in epilepsy of mesial temporal origin. Neurology 1990;40:1420-6.

27 Bartha L, Trinka E, Ortler $M$, et al. Linguistic deficits following left selective amygdalohippocampectomy: a prospective study. Epilepsy Behav 2004;5:348-57.
28 Delazer M, Gasperi A, Bartha L, et al. Number processing in temporal lobe epilepsy. J Neurol Neurosurg Psychiatry 2004;75:901-3.

29 Jokeit $H$, Ebner A. Effects of chronic epilepsy on intellectual functions. Prog Brain Res 2002; 135:455-63.

30 Lüders H, Lesser RP, Hahn J, et al. Basal temporal language area. Brain $1991 ; 114: 743-54$

31 Schäffler L, Lüders HO, Beck GJ. Quantitative comparison of language deficits produced by extraoperative electrical stimulation of Broca's, Wernicke's, and basal temporal language areas. Epilepsia 1996;37:463-75.

32 Krauss GL, Fisher R, Plate C, et al. Cognitive effects of resecting basal temporal language areas. Epilepsia 1996;37:476-83.

33 Murtha S, Chertkow $\mathrm{H}$, Beauregard $\mathrm{M}$, et al. The neural substrate of picture naming. J Cogn Neurosci 1999;1 1:399-423.

34 Bartha L, Brenneis C, Schocke M, et al. Medial temporal lobe activation during semantic language processing: fMRI findings in healthy left- and righthanders. Cogn Brain Res 2003;17:339-46.

35 Pihlajamäki $M$, Tanila $H$, Hanninen $T$, et al. Verbal fluency activates the left medial temporal lobe: a functional magnetic resonance imaging study. Ann Neurol 2000;47:470-6.

36 Gleissner U, Elger CE. The hippocampal contribution to verbal fluency in patients with temporal lobe epilepsy. Cortex 2001;37:55-63.

37 Brazdil M, Zakopcan J, Kuba R, et al. Atypical hemispheric language dominance in left temporal lobe epilepsy as a result of the reorganization of language functions. Epilepsy Behav 2003:4:414-9.

38 Knecht S, Drager B, Deppe $M$, et al. Handedness and hemispheric language dominance in healthy humans. Brain 2000;123:2512-8.

39 Springer JA, Binder JR, Hammeke TA, et al. Language dominance in neurologically normal and epilepsy subjects: a functional MRI study. Brain 1999;122:2033-46.

40 Tröster Al, Warmflash V, Osorio I, et al. The roles of semantic networks and search efficiency in verbal fluency performance in intractable temporal lobe epilepsy. Epilepsy Res 1995;21:19-26.

41 Hermann BP, Wyler AR, Richey ET. Wisconsin Card Sorting Test performance in patients with complex partial seizures of temporal-lobe origin. J Clin Exp Neuropsychol 1988;10:467-76.

42 Strauss E, Hunter M, Wada J. Wisconsin Card Sorting Performance: effects of age of onset of damage and laterality of dysfunction. J Clin Exp Neuropsychol 1993; 15:896-902.

43 Joanette $Y$, Goulet P. Criterion-specific reduction of verbal fluency in right brain-damaged right-handers. Neuropsychologia $1986: 24: 875-9$

44 Lifrak MD, Novelly RA. Language deficits in patients with temporal lobectomy for complex partial seizures. In: Porter RJ, Mattson RH, Ward AA, et al, eds. Advances in Epileptology: XVth Epilepsy International Symposium. New York: Raven Press, 1984:469-73.

45 Martin RC, Loring DW, Meador KJ, et al. The effects of lateralized temporal lobe dysfunction on formal and semantic word fluency. Neuropsychologia 1990;28:823-9.

46 Lehrl S, Triebig G, Fischer B. Multiple choice vocabulary test MWT as a valid and short test to estimate premorbid intelligence. Acta Neurol Scand 1995:91:335-45. 